\title{
Toward an improved hybrid model for simulating continuous flow microwave heating of water
}

\author{
G. Cuccurullo, L. Giordano \\ Department of Industrial Engineering \\ University of Salerno \\ Via Giovanni Paolo II n. 132, 84084 Fisciano, Italy \\ cuccuru@unisa.it, lagiordano@unisa.it
}

\author{
G. Viccione \\ Department of Civil Engineering \\ University of Salerno \\ Via Giovanni Paolo II n. 132, 84084 Fisciano, Italy \\ gviccion@unisa.it
}

\begin{abstract}
This paper introduces an improved hybrid (numerical-analytical) model for simulating microwave (MW) heating of laminar duct flow. The proposed procedure links numerical results to analytical calculations, providing a tool for accurate prediction of the bulk temperature distribution in a relatively reduced computation time, enhancing the design of MW heating of continuous flow water systems. The hybrid solution was obtained by first numerically solving the Maxwell equations in correspondence of an average dielectric permittivity; discrete values of the cross-section averaged heat generation arising from the numerical solution were first corrected by a suitable weighting function and then interpolated by a function resulting from the discrete Fourier series. The momentum and the energy equations fed by the above calculated heat generation distribution were uncoupled from Maxwell equations. The problem being linear, the analytical thermal solution was sought as the sum of two partial solutions, each one affected by a single non-homogeneity. The former solution turned out to be the classical Graetz problem, while the second one, driven by the heat generation, was solved in closed form by the variation of parameters method. On the other hand, the same problem was solved by a complete numerical approach in order to have a reference solution; thus, the Maxwell equations and the energy balance for the flowing fluid were simultaneously solved considering temperature dependent dielectric permittivity. Fully developed velocity, thermally developing conditions and no phase transition during the heating process were assumed for both the hybrid and the numerical solution. The availability of the reference solution allowed to prove the substantial enhancement of the hybrid solution in describing the bulk temperature distribution along the pipe when compared to the one related to the classical constant properties approach. Results, presented and discussed for different inlet velocities, show that increasing velocities provide a better agreement due to the smoothing effect realized by higher frequencies fluctuations in heat generation distribution felt by the flowing fluid.
\end{abstract}

Keywords-Microwave heating; hybrid models, Maxwell's equations; Comsol multiphysics

\section{INTRODUCTION}

Microwave (MW) heating has become a valuable and cost effective source of energy. Sure enough, volumetric heating of the target due to MWs, leads to higher heat rates and therefore to shorter processing times than those needed in traditional heating methods. In addition, when compared to traditional heating, MW heating exhibits quick startups, space and energy saving, and, not least, makes no environmental pollution. Such distinctive features have become more and more appealing both for household and industrial applications. Just to mention some glaring examples, because of the reduced thermal transients, as well as of the not excessively hot food surface compared with the inner side, the demand for domestic MW oven has constantly increased in recent years. In food industry, MWs have successfully employed for pasteurization, sterilization, etc. [1,2]. Other emerging applications concern the application of substrates to carbon nanotubes [3], ceramic [4] or metallic processing [5].

Recently, MWs are being utilized to heat continuous flow of liquids [6] and specifically, of water [7]. Related benefits concern the increased productivity, easier clean up and process automation compared to standard batch systems [8] in which the outward of the heat exchanger heats first and then heat is transferred to the inner liquid. Moreover, under laminar flow conditions, greater temperature increases and heat transfer rates can be attained [6], [9-10], compared to turbulent or transition regimes. This is not surprising if one considers that heat transfer is no more driven by the walls.

Despite all the cited benefits, still some disadvantages remain: the application of an external electric field produces an uneven temperature patterns in the samples which, in turn, are related to spatial variability of the electric field patterns [11]. A further major obstacle is related to difficulties in predicting the electromagnetic (EM) field; several approaches have been attempted to this purpose, mostly numerical and experimental. Only simplified analytical solutions are available due to the complexity of the problem at hand: in fact, thermal response is to be correlated to relative load and system configurations and to thermal and dielectric properties of the material as a function of chemical composition, temperature, and frequency [12-17]. With reference to the experimental approach, difficulties are experienced in measuring and/or controlling temperature because traditional probes fail and highly uneven temperature patterns are usually realized $[18,19]$. In addition, adequate experimentation may be impractical, as a large number of tests are usually required to obtain representative results $[20,21]$. Nowadays, the numerical approach allows a quite satisfying description of the coupled thermal-EM problems as well as an accurate identification of the effects which the operating parameters have on the process at hand [14, 17, 22-28], provided that spatial discretization is performed with care as 
grid dispersion may arise [6]. Starting from the pioneering work of Yee [29] in which Maxwell's equations were solved with a primitive 2D version of the Finite Difference Time Domain (FDTD) technique, remarkable contributions have been given so far. Zhang et al. [23] proposed a 3D FDTD model to describe electromagnetic field patterns, power temperature and velocity distributions in a confined liquid inside a microwave cavity. Chatterjee et al. [30] numerically analyzed the effects on the temperature distribution of a liquid in terms of the rotating container, natural convection, power sources and shape of the container. Zhu et al. [25], [31] developed a more sophisticated procedure to solve cases with temperature-dependent dielectric permittivity and nonNewtonian liquids carrying food subjected to MW heating. Actually, FDTDs [29] and Finite Element Methods (FEMs) [32] are no doubt among the most employed for simulating MW heating problems [33].

Numerical modeling may be subject to long execution times, depending on how complex is the system being simulated as well as on the spatial and temporal discretization. In the above connection, this paper proposes a hybrid numerical-analytical technique for simulating microwave (MW) heating of laminar flow in circular ducts, thus attempting to combine the benefits of analytical calculations and numerical field analysis methods, in order to deliver an approximate yet accurate prediction tool for the flow bulk temperature. The main novelty of the method relies on the combination of 3D FEM and analytical calculations, in an efficient thermal model, able to provide accurate results with moderate execution requirements. The proposed approach has successfully applied in similar contests $[34,35]$.

\section{Proposed Methodology}

\section{A. Hybrid Numerical-Analytical model definition}

The proposed methodology puts together 3D electromagnetic and thermal FEM results with analytical calculations, for the derivation of the temperature distribution for different flow rates. Numerical approach is used as an intermediate tool for calculating heat generation due to MW heating; the latter distribution, cross section averaged, allows to evaluate the $2 \mathrm{D}$ temperature distribution for the pipe flow by an analytical model in closed form. Such a procedure requires a sequential interaction of the analytical and numerical methods for thermal calculations, as illustrated in the flowchart of Fig. 1 and in the following described.

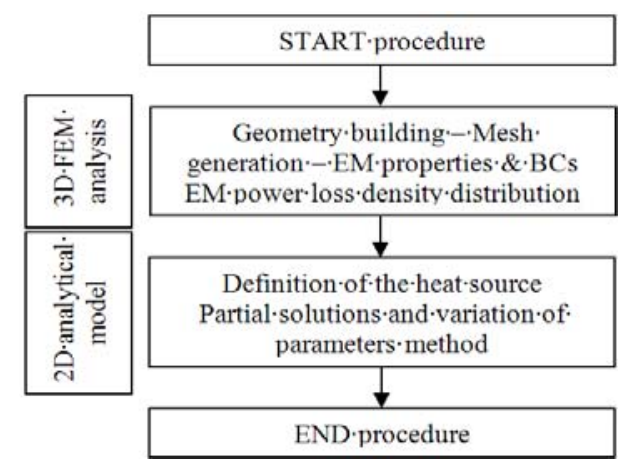

Fig.1. Flowchart of the assumed procedure
The developing temperature field for an incompressible laminar duct flow subjected to heat generation is considered. As first step, a 3D numerical FEM model was developed to predict the distribution of the EM field in water continuously flowing in a circular duct subjected to microwave heating. Water is described as an isotropic and homogeneous dielectric medium with electromagnetic properties independent of temperature. Maxwell's equations were solved in the frequency domain to describe the electromagnetic field configuration in the MW cavity supporting the applicator-pipe.

In view of the above hypotheses, the momentum and the energy equations turn out to be coupled through the heat generation term with Maxwell's equations. Then, an approximate analytical solution is obtained considering the effective heat generation distribution arising from the solution of the electromagnetic problem at hand to be replaced by its cross averaged section values; a further improved approximate analytical solution is obtained by considering a suitably weighting function for the heat dissipation distribution. In both cases the proper average value over the water control volume was retained by taking the one arising from the complete numerical solution. The possibility of recovering the fluid thermal behaviour by considering the two hybrid solutions is then investigated in the present work.

\section{B. 3D complete FEM Model description}

A general-purpose pilot plant producing microwaves by a magnetron rated at $2 \mathrm{~kW}$ and emitting at a frequency of $2.45 \mathrm{GHz}$ is available to the heat transfer laboratory, at the University of Salerno, Fig. 2; it will be used as reference for future developments of the present work, aiming to validate the results herein presented. Thus, the following models are referred to such an experimental setup.

The pipe carrying water to be heated was $8 \mathrm{~mm}$ internal diameter and $0.90 \mathrm{~m}$ long. Symmetrical geometry and load conditions about the XY symmetry plane are provided. Such a choice was performed having in mind to suitably reduce both computational burdens and mesh size while preserving the main aim of the paper that is to compare the two hybrid approximate analytical solution with the numerical one acting as reference. In particular, a cubic cavity chamber (side length, $L=0.9 \mathrm{~m}$ ) and a standard WR340 waveguide were assumed.

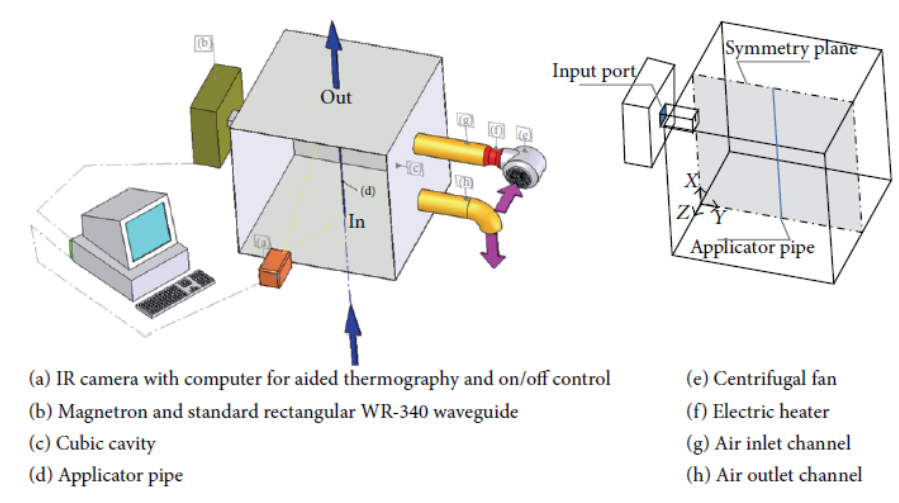

Fig.2. Sketch of the available setup 
The insulated metallic cubic chamber houses one PTFE applicator pipe allowing water continuous flow; the pipe is embedded in a box made by a closed-cell polymer foam, assumed to be transparent to microwaves at $2.45 \mathrm{GHz}$.

A 3D numerical FEM model of the above was developed by employing the commercial code COMSOL v4.3 [36]. It allows coupling electromagnetism, fluid, and energy flow to predict temperature patterns in the fluid continuously heated in a multimode microwave illuminated chamber. The need of considering coupled physics and thus a complete numerical solution $(\mathrm{CN})$, arises by noting that, due to the geometry at hand, no simplified heating distributions can be sought (i.e. the ones based on Lambert Law's) [17]. Ruling equations are solved by means of the finite element method (FEM) using unstructured tetrahedral grid cells.

The electric field distribution $\underline{E}$ in the microwave cavity, both for air and for the applicator pipe carrying the fluid under process, is determined by imposing

$$
\nabla \times\left(\frac{1}{\mu_{\mathrm{r}}} \nabla \times \underline{E}\right)-k_{0}^{2}\left(\varepsilon_{\mathrm{r}}-\frac{\mathrm{i} \sigma}{\omega \varepsilon_{0}}\right) \underline{E}=\underline{0}
$$

in which $\varepsilon_{\mathrm{r}}$ is the relative permittivity, $\omega$ is the angular wave frequency, $\mu_{\mathrm{r}}$ is the relative permeability of the material, $k_{0}$ is the wavenumber in vacuum, and $\sigma$ is the electric conductivity.

Air and the PTFE applicator tube were both supposed to be completely transparent to microwaves. Assuming negligible resistive material losses, boundary conditions for the radio frequency module included perfect electric conductors walls, that is, $\underline{n} \times \underline{E}=\underline{0}$, for the waveguide and the cavity, being $\underline{n}$ the local normal vector. At the port, an amount of $2 \mathrm{~kW}$ EM power, $2450 \mathrm{MHz}$ frequency, was supplied through a rectangular TE10 mode waveguide (WR 340).

Continuity boundary condition was set by default for all the interfaces between the confining domains, that is, the pipe, the cavity, and the waveguide. Such condition may be stated as

$$
\underline{n} \times\left(\underline{E}_{\mathrm{i}}-\underline{E}_{\mathrm{j}}\right)=\underline{0}
$$

being $i$ and $j$ the neighbouring discrete portions sharing the interface at hand. Scattering boundary conditions were applied at the inlet and the outlet of the pipe to make the pipe's ends transparent to incoming waves, avoiding that undesired reflected waves travel inward [20].

Due to the symmetry of the problem, and load conditions around the XY plane crossing vertically the oven, the waveguide, and the pipe (see Fig. 1, right side) the model is reduced to one-half of the device, yielding a more accuracy in the calculation. The condition of perfect magnetic conductor was applied for the surfaces yielding on the symmetry plane:

$$
\underline{n} \times \underline{H}=\underline{0}
$$

$\underline{H}$ being the magnetic field, which has to be therefore parallel to the local normal vector $\underline{n}$ on the XY plane.
Temperature distribution is determined for fully developed Newtonian fluid in laminar motion, considering constant flow properties; in such hypotheses, the energy balance reduces to

$$
\rho c_{\mathrm{p}} U \frac{\partial T}{\partial X}=k \nabla^{2} T+U_{\mathrm{gen}}
$$

where $T$ is the temperature, $\rho$ is the fluid density, $c_{\mathrm{p}}$ is the specific heat, $k$ is the thermal conductivity, $X$ is the axial coordinate, $U(R)=2 U_{\text {av }}\left(1-4 \cdot \mathrm{R}^{2} / \mathrm{D}_{\mathrm{i}}^{2}\right)$ is the axial Poiseille velocity profile, $D_{\mathrm{i}}$ being the internal pipe diameter and $R$ the radial coordinate; $U_{\text {gen }}$ is the specific heat generation, i.e. the "electromagnetic power loss density" $\left(\mathrm{W} / \mathrm{m}^{3}\right)$ resulting from the EM problem. The power-generation term realizes the coupling of the EM field with the energy balance equation where it represents the "heat source" term:

$$
U_{\mathrm{gen}}(X, Y, Z)=\frac{1}{2} \omega \varepsilon_{0} \varepsilon^{\prime \prime}|\underline{E(X, Y, Z)}|^{2}
$$

where $\varepsilon_{0}$ is the free-space permittivity and $\varepsilon$ " is the relative dielectric loss of the material.

The two-way coupling arises by considering temperature dependent dielectric permittivity. Such dependence is expressed by a polynomial approx.:

$$
\begin{gathered}
\varepsilon^{\prime}(T)=-425.963+5.16708 \cdot T-0.0167823 \cdot T^{2}+ \\
0.0000171415 \cdot T^{3}
\end{gathered}
$$

$\varepsilon "(T)=1435.84-12.2473 \cdot T+0.0350158 \cdot T^{2}-0.0000334891 \cdot T^{3}$

which is based on data by [37].

\section{The hybrid solution}

\section{C.1. The heat generation definition}

In this case, the Maxwell's equations are solved first by considering a fixed, temperature independent, dielectric permittivity value. Both the real and imaginary part of the permittivity are selected by evaluating (6) and (7) in correspondence of the arithmetic average temperature $T_{\text {avg }}$ arising from the complete numerical solution described in section B. Such a move allows to uncouple the thermal and the EM sub-problems: the power-generation term realizes the oneway coupling of the EM field with the energy balance equation. Considering that the internal pipe diameter is much lower than the pipe length, a simplified cross averaged distribution is sought: its cross averaged value is selected instead, $U_{\text {gen }}(\mathrm{X})$.

A first basic hybrid solution, $\mathrm{BH}$, is obtained by rescaling the $U_{\text {gen }}(\mathrm{X})$ distribution so to retain the overall energy, $U_{0} V$, as resulting from integration of (5) over the entire water volume, $V$ : 


$$
\hat{U}_{\text {gen }, \mathrm{BH}}(X)=\hat{U}_{\text {gen }}(X) \cdot \frac{U_{0}}{\hat{U}_{\text {gen }_{\text {avg }}}}
$$

A further enhanced hybrid solution, EH, is obtained by first weighting and then rescaling $U_{\text {gen }}(X)$. In the light of (5), the weighting function is selected as:

$$
W(X)=\frac{\varepsilon^{\prime \prime}\left[T_{b}(X)\right]}{\varepsilon^{\prime \prime}\left[T_{\mathrm{b}, \mathrm{avg}}\right]}
$$

where $\mathrm{T}_{b}(X)$ is the bulk temperature corresponding the limiting case of uniform heat generation, $U_{0}$. Finally, the heat dissipation rate for the $\mathrm{EH}$ solution is obtained:

$$
\hat{U}_{\mathrm{gen}, \mathrm{EH}}(X)=\hat{U}_{\mathrm{gen}}(X) \cdot W(X) \cdot \mathrm{U}_{0}^{\prime}
$$

where $\mathrm{U}_{0}^{\prime}$ forces the overall energy to be $U_{0} V$. Consider that, in practice, the parameter $U_{0}$ can be measured by calorimetric methods, therefore enabling the application of the analytical model with ease. In Fig. 3 the two different heat generation distributions for the $\mathrm{BH}$ and $\mathrm{EH}$ problems are reported and compared with the cross section averaged values corresponding to the $\mathrm{CN}$ solution. Plots are referred to an arbitrarily selected $U_{a v}$ which determines the bulk temperature level of the pipe applicator, $\mathrm{T}_{\mathrm{b} \text {,avg. }}$. The $\mathrm{CN}$-curve is practically overlapped to the EH-curve, thus showing a major improvement with respect to the $\mathrm{BH}$-curve.

\section{C.2. The $2 D$ analytical model}

The thermal model provides laminar thermally developing flow of a Newtonian fluid with constant properties and negligible axial conduction. In such hypotheses, the dimensionless energy balance equation and the boundary conditions in the thermal entrance region turn out to be:

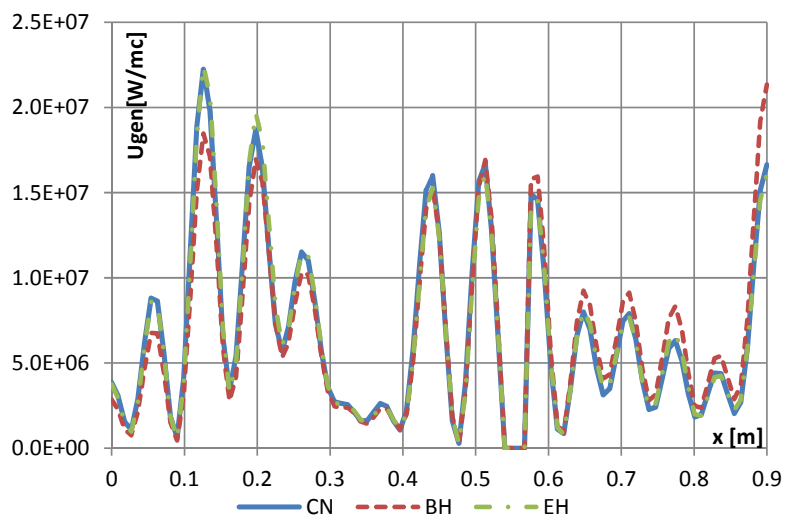

Fig 3. Heat generation along the $X$ axis for $U_{a v}=0.08 \mathrm{~m} / \mathrm{s}$

$$
2 \cdot\left(1-r^{2}\right) \cdot \frac{\partial t}{\partial x}=\frac{1}{r} \cdot \frac{\partial}{\partial r}\left(r \cdot \frac{\partial t}{\partial r}\right)+u_{\mathrm{gen}, \mathrm{H}}
$$

$$
\left.\frac{\partial t}{\partial r}\right|_{r=1}=0
$$

$$
\left.\frac{\partial t}{\partial r}\right|_{r=0}=0
$$

$$
t(0, r)=1
$$

where $t=\left(T-T_{\mathrm{s}}\right) /\left(T_{\mathrm{i}}-T_{\mathrm{s}}\right)$ is the dimensionless temperature, being $T_{\mathrm{s}}$ and $T_{\mathrm{i}}$ the temperature of the ambient surrounding the tube and the inlet flow temperature, respectively; $X$ and $R$ are the axial and radial coordinate; thus, $x=(4 \cdot X) /\left(P e \cdot D_{\mathrm{i}}\right)$ is the dimensionless axial coordinate, with the Peclet number defined as: $P e=\left(U_{\mathrm{av}} \cdot D_{\mathrm{i}}\right) / \alpha$, being $\alpha$ the thermal diffusivity, $r=(2 \cdot R) / D_{\mathrm{i}}$ is the dimensionless radial coordinate; $u_{\mathrm{gen}, \mathrm{H}}=$ $\left(U_{\text {gen, } \mathrm{H}} \cdot D_{\mathrm{i}}{ }^{2}\right) /\left(4 \cdot k \cdot\left(T_{\mathrm{i}}-T_{\mathrm{s}}\right)\right)$ is the dimensionless hybrid heat generation level, being $U_{\text {gen,H }}$ the corrected heat generation distribution alternatively given by (8) or (10), $k$ the thermal conductivity.

The two $\mathrm{BH}$ and $\mathrm{EH}$ heat generation distributions obtained in the previous section were turned into continuous interpolating function by using the Discrete Fourier Transform:

$$
\frac{u_{\text {gen }, \mathrm{H}}(x)}{k_{1}}=1+\sum_{\mathrm{n}=1}^{\mathrm{N} / 2}\left[\beta_{\mathrm{n}} \operatorname{Sin}(n \omega x)+\gamma_{\mathrm{n}} \operatorname{Cos}(n \omega x)\right]
$$

where: $k_{1}=\left(U_{0} \cdot D_{\mathrm{i}}^{2}\right) /\left(4 \cdot k \cdot\left(T_{\mathrm{i}}-T_{\mathrm{s}}\right)\right), \beta_{\mathrm{n}}=B_{\mathrm{n}} / U_{0}$ and $\gamma_{\mathrm{n}}=G_{\mathrm{n}} / U_{0}, B_{\mathrm{n}}$ and $G_{\mathrm{n}}$ being the magnitudes of the Sine a Cosine functions; $\omega$ is related to the fundamental frequency and $\mathrm{N}$ is the number of the discrete heat generation values. The expression (15) for the heat generation was used to solve the set of (11)-(14).

The resulting problem being linear, the thermal solution has been written as the sum of two partial solutions:

$$
t(x, r)=t_{\mathrm{G}}(x, r)+k_{1} \cdot t_{\mathrm{V}}(x, r)
$$

The function $t_{\mathrm{G}}(x, r)$ represents the solution of the extended Graetz problem featured by a nonhomogeneous equation at the inlet and adiabatic boundary condition at wall. On the other hand, the function $t_{\mathrm{V}}(x, r)$ takes into account the microwave heat dissipation and exhibits a non-homogeneity in the differential equation. Thus, the two partial solutions have to satisfy the two distinct problems respectively reported in the set (17)-(20) and (21)-(24). 


$$
\begin{aligned}
& \left\{\begin{array}{l}
2 \cdot\left(1-r^{2}\right) \frac{\partial t_{G}}{\partial x}=\frac{1}{r} \frac{\partial}{\partial r}\left(r \frac{\partial t_{G}}{\partial r}\right) \\
\left.\frac{\partial t_{G}}{\partial r}\right|_{\mathrm{r}=1}=0 \\
\left.\frac{\partial t_{G}}{\partial r}\right|_{\mathrm{r}=0}=0 \\
t_{G}(0, r)=1
\end{array}\right. \\
& \left\{\begin{array}{l}
2 \cdot\left(1-r^{2}\right) \frac{\partial t_{\mathrm{V}}}{\partial x}=\frac{1}{r} \frac{\partial}{\partial r}\left(r \frac{\partial t_{\mathrm{V}}}{\partial r}\right)+1+ \\
+\sum_{i=1}^{N / 2}\left[\beta_{n} \sin (n \omega x)+\gamma_{n} \cos (n \omega x)\right] \\
\left.\frac{\partial t_{\mathrm{V}}}{\partial r}\right|_{r=1}=0 \\
\left.\frac{\partial t_{\mathrm{V}}}{\partial r}\right|_{\mathrm{r}=0}=0 \\
t_{V}(0, r)=0
\end{array}\right.
\end{aligned}
$$

The Graetz problem was solved in closed form through the separation variables method, so the structure of the solution was the following:

$$
t_{\mathrm{G}}(x, r)=\sum_{\mathrm{m}=1}^{\mathrm{M}} c_{\mathrm{m}} F_{\mathrm{m}}(r) \operatorname{Exp}\left[-\frac{\lambda_{\mathrm{m}}^{2}}{2} \cdot x\right]
$$

where

$$
\mathrm{F}_{\mathrm{m}}(\mathrm{r})=\mathrm{e}^{-\frac{\mathrm{r}^{2} \cdot \lambda_{m}}{2}} \cdot \mathrm{La}\left[\frac{1}{4}\left(-2+\lambda_{m}\right), \mathrm{r}^{2} \cdot \lambda_{m}\right]
$$

are the eigen-functions, $L a$ being the orthonormal Laguerre polynomials, and $\lambda_{\mathrm{m}}$ are the related eigenvalues arising from the characteristic equation: $F_{\mathrm{m}}{ }^{\prime}(1)=0$.

The " $\mathrm{t}_{\mathrm{v}}$ " problem was solved in closed form by the variation of parameters method which allows to find the solution of a linear but non homogeneous problem even if the $x$-stationary solution doesn't exist. The solution was sought as:

$$
t_{\mathrm{V}}(x, r)=\sum_{\mathrm{j}=1}^{J} A_{\mathrm{j}}(x) \cdot F_{\mathrm{j}}(r)
$$

where $F_{\mathrm{j}}(r)$ are the eigen-functions of the equivalent homogeneous problem (obtained from the " $t_{\mathrm{V}}$ " problem by deleting the generation term) and are equal to the Graetz problem ones.
The orthogonality of the eigen-functions respect to the weight $r \cdot\left(1-r^{2}\right)$ allowed to obtain the following fist order differential equation, which satisfies both the " $t_{\mathrm{V}}$ " differential equation and its two " $\mathrm{r}$ " boundary conditions:

$$
\frac{d A_{\mathrm{j}}(x)}{d x}+\frac{1}{2} \lambda_{\mathrm{j}}^{2} A_{\mathrm{j}}(x)=f(x) \frac{H_{\mathrm{j}}}{E_{\mathrm{j}}}
$$

where

$$
\begin{gathered}
E_{\mathrm{j}}=\int_{0}^{1} F_{\mathrm{j}}^{2}(r) r\left(1-r^{2}\right) d r \\
H_{\mathrm{j}}=\int_{0}^{1} \frac{1}{2} r F_{\mathrm{j}}(r) d r \\
f(x)=1+\sum_{\mathrm{i}=1}^{\mathrm{N} / 2}\left[\beta_{\mathrm{n}} \sin (n \omega x)+\gamma_{\mathrm{n}} \cos (n \omega x)\right]
\end{gathered}
$$

Equation (28) was solved imposing the " $x$ " boundary condition of the " $\mathrm{v}_{\mathrm{v}}$ " problem, which in terms of $A_{\mathrm{j}}(x)$ turns out to be:

$$
A_{\mathrm{j}}(0)=0
$$

In particular, the linearity of the problem suggested to find the functions $A_{\mathrm{j}}(x)$ as the sum of $N / 2$ - partial solutions, each one resulting from a simple differential partial equation correlated with the boundary condition:

$$
a_{\mathrm{ji}}{ }^{\prime}(x)+\frac{1}{2} \cdot \lambda_{\mathrm{j}}^{2} \cdot a_{\mathrm{ji}}(x)=\frac{H_{\mathrm{j}}}{E_{\mathrm{j}}}, \quad l=1
$$

$a_{\mathrm{ji}}^{\prime}(x)+\frac{\lambda_{\mathrm{j}}^{2} a_{\mathrm{ji}}(x)}{2}=\frac{H_{\mathrm{j}}}{E_{\mathrm{j}}}\left[\beta_{\mathrm{n}} \sin (n \omega x)+\gamma_{\mathrm{n}} \cos (n \omega x)\right]$

where $i=2 \ldots N / 2$. Finally,

$$
a_{\mathrm{ji}}(0)=0
$$

Then, for a fixed value of $j$, the function $A_{\mathrm{j}}(x)$ turns out to be:

$$
A_{\mathrm{j}}(x)=\sum_{i=1}^{N / 2} a_{\mathrm{ji}}(x)
$$

Finally, it was verified that such an analytical solution recovers the corresponding numerical results. 


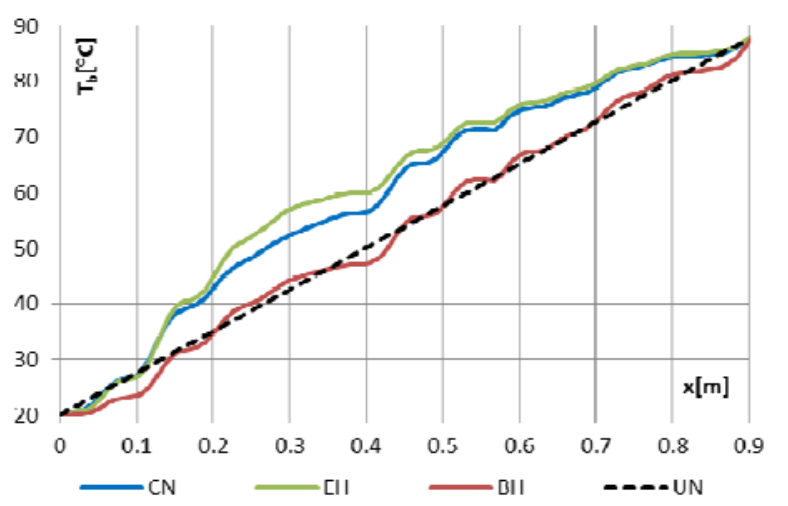

Fig. 4. Bulk temperature evolution for $U_{a v}=0.008 \mathrm{~m} / \mathrm{s}$

\section{RESULTS}

\section{A. Bulk temperature analysis}

Bulk temperature distributions are plotted in Figs. 4 and 5 for two different inlet velocities, namely 0.008 and $0.08 \mathrm{~m} / \mathrm{s}$. Curves are related to the $\mathrm{CN}, \mathrm{EH}, \mathrm{BH}$ problems and, for reference, a further one evaluated analytically assuming uniform $U_{0}$ heat generation (UN). It clearly appears that the $\mathrm{EH}$ problem fits quite well the $\mathrm{CN}$ problem, whereas the remaining curves underestimate it. In particular, $\mathrm{EH}$ and $\mathrm{CN}$ curves are almost overlapped for the highest velocity.

In order to quantitatively compare results, the root mean square error RMSE $\left[{ }^{\circ} \mathrm{C}\right]$ with respect to the $\mathrm{CN}$ solution is evaluated by considering a sampling rate of 10 points per wavelength, Fig.6. For a fixed $\mathrm{U}_{\mathrm{av}}$, the RMSE related to the $\mathrm{UN}$ and $\mathrm{BH}$ curves are practically the same since the $\mathrm{BH}$ curve fluctuates around the dashed one, whereas the corresponding the $\mathrm{EH}$ values turn out to be noticeably reduced.

Interestingly enough, the more is the inlet velocity, the lower is the RMSE, providing a better agreement due to the smoothing effect realized by higher frequencies fluctuations in heat generation felt by the flowing fluid.

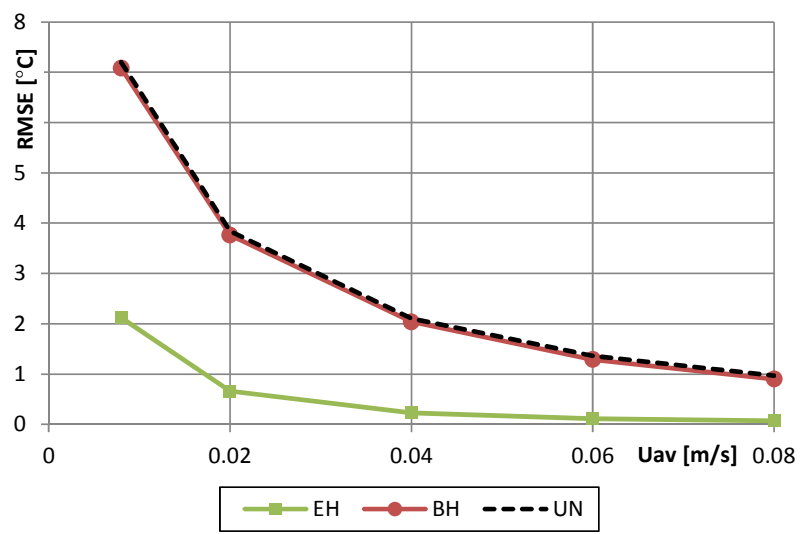

Fig. 6. Root mean square error with respect to the $\mathrm{CN}$ solution

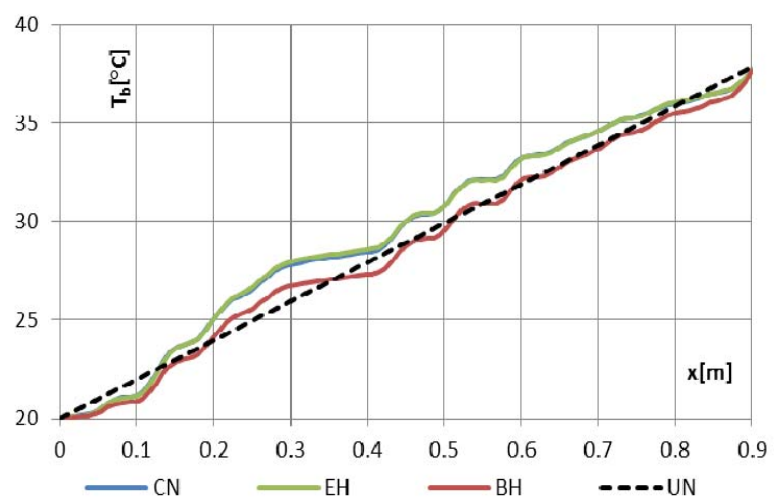

Fig. 5. Bulk temperature evolution for $U_{a v}=0.08 \mathrm{~m} / \mathrm{s}$

\section{B. Cross sections of temperature spatial distribution}

In order to have a birds-eye view over the cross section temperature distribution, two different pipe cross sections corresponding to $U_{a v}=0.008$ and $0.08 \mathrm{~m} / \mathrm{s}$ are investigated. Sections were chosen such as the relative normalized error computed with respect to the $\mathrm{CN}$ solution attained an arbitrarily fixed value of 0.1 . In the light of what observed about the bulk temperature behavior in dependence of $U_{a v}$, the two sections resulted to be located at 737 and $533 \mathrm{~mm}$ from the inlet for $\mathrm{U}_{\mathrm{av}}=0.008$ and $0.08 \mathrm{~m} / \mathrm{s}$ respectively.

Fig. 7 provides contour plots related to temperature differences between $\mathrm{CN}$ and $\mathrm{EH}$ models for the two above identified sections. As can be seen, the case $U_{a v}=0.08 \mathrm{~m} / \mathrm{s}$ yields greater discrepancies in the spatial temperature distribution, even if such differences are confined in a small region nearby the pipe wall. Maximum difference is equal to $8^{\circ} \mathrm{C}$ (EH solution locally overestimated) for such case. For the case $U_{a v}=0.008 \mathrm{~m} / \mathrm{s}$ there are lower discrepancies in the temperature fields: the maximum difference is equal to $+3^{\circ} \mathrm{C}$ (EH solution locally underestimated). It is expected that such differences decrease with increasing pipe length since increased fluctuations number facilitates the stabilisation of the local average value.

\section{CONCLUSIONS}

In the following, bulk temperature distributions The process of continuous flow microwave heating was simulated both using a multi-physics software package and two approximated numerical-analytical hybrid models, encompassing a longitudinal variable heat dissipation inside the entrance region of the heated pipe. The discrete values of the heat generation arising from the numerical solution were interpolated by a function resulting from the Discrete Fourier Series in order to fed the analytically solved thermal problem.

The use of a properly stretched heat generation distribution allowed to describe the bulk temperature distribution much better than the corresponding problem featured by constant EM properties. Furthermore, such an aim is recovered with no additional computational efforts, thus leading to an easy way to predict temperature patterns through the pipe. Further 


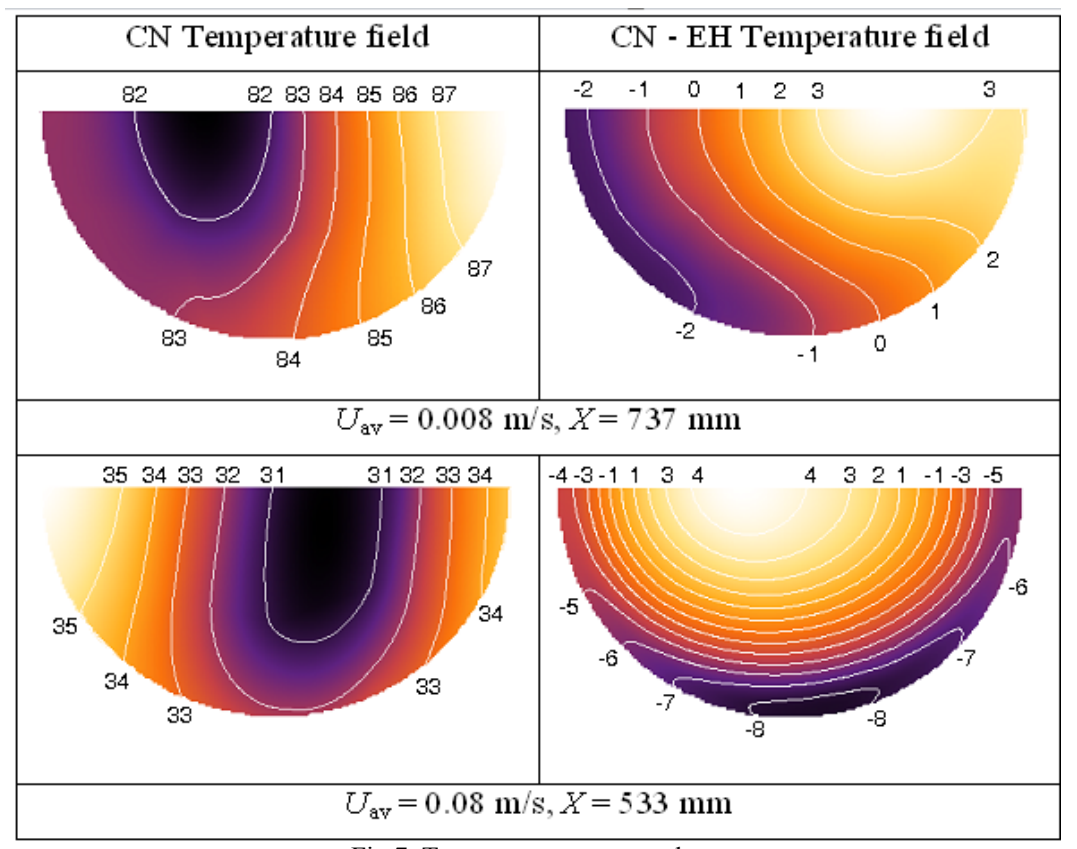

Fig.7. Temperature contour plots

developments of the present work are intended to validate the present results by means of experimental investigations.

\section{REFERENCES}

[1] I. Sierra, C. Vidal-Valverde, and A. Olano, "The effects of continuous flowmicrowave treatment and conventional heating on the nutritional value of milk as shown by influence on vitamin B1 retention," European Food Research and Technology, vol. 209, no. 5, pp. 352-354, 1999.

[2] S. Tajchakavit, H. S. Ramaswamy, and P. Fustier, "Enhanced destruction of spoilage microorganisms in apple juice during continuous flow microwave heating," Food Research International, vol. 31, no. 10, pp. 713-722, 1998.

[3] H.-C. Su, C.-H. Chen, , Y.-C. Chen, D.-J. Yao, H. Chen, Y.-C. Chang, and T.-R. Ywe, "Improving the Adhesion of Carbon Nanotubes to a Substrate Using Microwave Treatment,". Carbon 2010, 48, 805-812.

[4] S. Das, A. K. Mukhopadhyay, S. Datta, and D. Basu, "Prospects of microwave processing: An overview," Bull. Mater. Sci., Vol. 32, No. 1, February 2009, pp. 1-13.

[5] D. Agrawal, "Latest global developments in microwave materials processing," Materials Research Innovations, Volume 14, Number 1, February 2010 , pp. 3-8(6).

[6] G. Cuccurullo, L. Giordano, and G. Viccione, "An Analytical Approximation for Continuous Flow Microwave Heating of Liquids," Advances in Mechanical Engineering Volume 2013, 8 pages, http://dx.doi.org/10.1155/2013/929236

[7] T. Yousefi, S.A. Mousavi, M.Z. Saghir, and B. Farahbakhsh, "An investigation on the microwave heating of flowing water: A numerical study," International Journal of Thermal Sciences (2013), http://dx.doi.org/10.1016/j.ijthermalsci.2013.04.006

[8] J. Ahmed and S. Hosahalli Ramaswamy, "Microwave pasteurization and sterilization of foods," in Handbook of Food Preservation, M. Shafiur Rahman, Ed., chapter 28, pp. 691-711, 2nd edition, 2007.

[9] G. Cuccurullo and V. Spingi, "An approximate solution for the entrance region in laminar pipe flow with temperature dependent heat generation," in Proceedings of the 29th UIT Heat Transfer Conference, Torino, Italy, June 2011.

[10] G. Cuccurullo, L. Giordano, V. Spingi, F. D’Agostino, M. Migliozzi, “A numerical-analytical solution for continuous flow microwave heating of liquids in laminar motion," in Proceedings of the 30th UIT Heat Transfer Conference, Bologna, Italy, June 2012.

[11] K.G. Ayappa, H.T. Davis, E.A. Davis, J. Gordon, "Analysis of microwave heating of materials with temperature-dependent properties," AIChE Journal 37 (1991) 313-322.

[12] R. Vadivambal and D. S. Jayas, "Non-uniform temperature distribution during microwave heating of food materials-a review," Food and Bioprocess Technology, vol. 3, no. 2, pp. 161-171, 2010.

[13] P. Coronel, J. Simunovic, and K. P. Sandeep, "Temperature profiles within milk after heating in a continuous-flow tubular microwave system operating at 915MHz," Journal of Food Science, vol. 68, no. 6, pp. 1976-1981, 2003.

[14] D. Salvi, J. Ortego, C. Arauz, C. M. Sabliov, and D. Boldor, "Experimental study of the effect of dielectric and physical properties on temperature distribution in fluids during continuous flow microwave heating," Journal of Food Engineering, vol. 93, no. 2, pp. 149-157, 2009.

[15] N. M. Gerbo, D. Boldor, and C. M. Sabliov, "Design of a measurement system for temperature distribution in continuousflow microwave heating of pumpable fluids using infrared imaging and fiber optic technology," Journal of Microwave Power and Electromagnetic Energy, vol. 42, no. 1, pp. 55-65, 2008.

[16] C. M. Sabliov, D. A. Salvi, and D. Boldor, "High frequency electromagnetism, heat transfer and fluid flow coupling in ANSYS multiphysics," Journal of Microwave Power and Electromagnetic Energy, vol. 41, no. 4, pp. 5-17, 2007.

[17] A. Datta, H. Prosetya, and W. Hu, "Mathematical modeling of batch heating of liquids in a microwave cavity," Journal of Microwave Power and Electromagnetic Energy, vol. 27, no. 1, pp. 38-48, 1992.

[18] G. Cuccurullo, L. Cinquanta, and G. Sorrentino, "A procedure to achieve fine control in MW processing of foods," Infrared Physics and Technology, vol. 49, no. 3, pp. 292-296, 2007.

[19] G. Cuccurullo, L. Giordano, D. Albanese, L. Cinquanta, andM. Di Matteo, "Infrared thermography assisted control for apples microwave drying," Journal of Food Engineering, vol. 112, pp.319-325, 2012.

[20] P. D. Muley and D. Boldor, "Multiphysics numerical modeling of the continuous flow microwave-assisted transesterification process," Journal of Microwave Power and Electromagnetic Energy, vol. 46, no. 3, pp. 139-162, 2012. 
[21] K. Knoerzer, M. Regier, and H. Schubert, "Microwave heating: a new approach of simulation and validation," Chemical Engineering and Technology, vol. 29, no. 7, pp. 796-801, 2006.

[22] D. Salvi, D. Boldor, G. M. Aita, and C. M. Sabliov, "COMSOL Multiphysics model for continuous flow microwave heating of liquids," Journal of Food Engineering, vol. 104, no. 3,pp. 422-429, 2011.

[23] Q. Zhang, T.H. Jackson, andA.Ungan, "Numerical modeling of microwave induced natural convection," International Journal of Heat and Mass Transfer, vol. 43, pp. 2141-2154, 2000.

[24] C. Mirabito, A. Narayanan, D. Perez, and B. Stone, FEMLAB Model of a Coupled Electromagnetic-Thermal Boundary Value Problem. Research Experience, Worcester Polytechnic Institute, Worcester, Mass, USA, 2005.

[25] J. Zhu, A. V. Kuznetsov, and K. P. Sandeep, "Mathematical modeling of continuous flow microwave heating of liquids (effects of dielectric properties and design parameters)," International Journal ofThermal Sciences, vol. 46, no. 4, pp. 328-341, 2007.

[26] P. Ratanadecho, K. Aoki, and M. Akahori, "A numerical and experimental investigation of the modeling of microwave heating for liquid layers using a rectangular wave guide (effects of natural convection and dielectric properties)," Applied Mathematical Modelling, vol. 26, no. 3, pp. 449-472, 2002.

[27] A. Le Bail, T. Koutchma, and H. S. Ramaswamy, "Modeling of temperature profiles under continuous tube-flow microwave and steamheating conditions," Journal of Food Process Engineering, vol. 23, pp. 1-24, 2000.

[28] D. A. Salvi, D. Boldor, C.M. Sabliov, and K. A. Rusch, "Numerical and experimental analysis of continuous microwave heating of ballast water as preventive treatment for introduction of invasive species," Journal of Marine Environmental Engineering, vol. 9, no. 1, pp. 45-64, 2008.

[29] K.S. Yee, "Numerical solution of initial boundary value problem involving Maxwell's equations in isotropic media," IEEE Transactions on Antennas and Propagation 14 (1966) 302-307.

[30] S. Chatterjee, T. Basak, S.K. Das, Microwave driven convection in a rotating cylindrical cavity: a numerical study, Journal of Food Engineering 79 (2007) 1269-1279.

[31] J. Zhu, A. V. Kuznetsov, and K. P. Sandeep, "Investigation of a particulate flow containing spherical particles subjected to microwave heating," Heat and Mass Transfer, vol. 44, no. 4, pp. 481-493, 2008.

[32] O. C. Zienkiewicz, R. L. Taylor, and J. Z. Zhu, The Finite Element Method: Its Basis and Fundamentals, Butterworth-Heinemann, 6th edition, 2005.

[33] V.V. Yakovlev, Examination of contemporary electromagnetic software capable of modeling problems of microwave heating, in: M. WillertPorada (Ed.), Advances in Microwave and Radio Frequency Processing, Springer Verlag, 2005, pp. 178-190.

[34] M. A. Tsili, E. I. Amoiralis, A. G. Kladas, and A. T. Souflari, "Hybrid Numerical-Analytical Technique for Power Transformer Thermal Modeling," IEEE Transactions on Magnetics, Vol. 45, no. 3, march 2009.

[35] R. M. Cotta, "Hybrid Numerical/Analytical approach to nonlinear diffusion problems," Numerical Heat Transfer, Part B: Fundamentals: An International Journal of Computation and Methodology Vol. 17(2), 1990, 217-226.

[36] COMSOL Multiphysics Version 4.3a User Guide, October 2012.

[37] W. J. Ellison, "Permittivity of Pure Water, at Standard Atmospheric Pressure, over theFrequency Range $0-25 \mathrm{THz}$ and the Temperature Range 0-100 'C", J. Phys. Chem. Ref. Data, Vol. 36, No. 1, 2007.

\section{Creative Commons Attribution License 4.0 (Attribution 4.0 International, CC BY 4.0)}

This article is published under the terms of the Creative Commons Attribution License 4.0 https://creativecommons.org/licenses/by/4.0/deed.en US 